\title{
COMET watching
}

\section{Derek Richards}

Editor, Evidence-based Dentistry

In November 1998 we published the first issues of this journal as a supplement to the BDJ and in the same years the NASA NearEarth Object Program Office (NEOPO) was established at the Jet Propulsion Laboratory. Since then there has been significant increase in our knowledge about asteroids and comets and on $12^{\text {th }}$ November the European Space Agency's Rosetta mission is hoping to land on the surface of comet 67P/ Churyumov-Gerasimenko.

The huge amount of information on asteroids and comets generated by NEOPO is mirrored by the explosion in the number of journals and volume of health sciences literature that are released each year. For example Pubmed now has more than 24 million articles in its database, and this database only includes a selection of the available healthcare journals. This increase in the number of available articles has increased the value and utility of the review as a means of summarising the available knowledge on a given topic. However the limitations of the traditional review were exposed in the 1980s, ${ }^{1,2}$ and the need for review with a rigorous and systematic approach recognised. The basic steps of systematic reviewing are:

1. Define the question

2. Search the literature

3. Assess the studies identified

4. Analyse and summarise the data

5. Place the findings in context.

Detailed guidance on how to prepare a systematic review is available on line..$^{3,4}$ Systematic reviews were originally developed to assess the effectiveness of clinical interventions but they are now being used to bring together a broader range of literature including observational and diagnostic studies. Summarising systematic reviews is core to what we do here in the EBD journal and there are a number of common issues that arise:

1. a limited number of studies are available to address a particular issue
2. the quality of the available studies is poor or inadequate to answer the question

3. the outcomes measures employed by the researchers are too variable to easily combine.

I have written previously about the need to improve the quality of dental research, ${ }^{5}$ and developments such as the Equator (Enhancing the QUAlity and Transparency Of health Research) network, who now have an extensive library of health reporting guidelines on their website, (http:// www.equator-network.org/) have done much to highlight what key elements of studies should be reported. These guidelines also form a useful resource for researchers planning a new project, as these guidelines highlight key methodological issues that need to be addressed for a range of different study designs. By paying attention to these guidelines researchers address the first two bullet points noted above and improve the chances of getting their research published.

Addressing the third bullet point of the variability in the range of outcome measures in dental studies is another challenge. This has long been a problem and a 2009 review by Savage et al. ${ }^{6}$ highlighted the issues in periodontal diseases. This is an issue that is not just pertinent to oral health research but healthcare research in general and it is to try and address this problem that The COMET (Core Outcome Measures in Effectiveness Trials) Initiative was launched in Liverpool in 2010.

The COMET initiative brings together researchers interested in developing and applying agreed standardised sets of outcomes, known as a 'core outcome set.'

Core outcome sets (COS) represent the minimum that should be measured and reported in all clinical trials of a specific condition. Having a minimum recordable dataset should make it easier for trial results to be compared and combined as appropriate. This in time should improve the information available from reviews. It is not the intention to restrict datasets to the relevant COS but to explore other outcomes in addition.

A website has been developed to support the COMET Initative (http://www.cometinitiative.org/). This includes a database of relevant resources that incudes useful references for COS developers and information about ongoing projects. Currently it lists some 22 entries for dentistry and oral health including four COS consensus projects for

1. Selective outcome reporting in randomised controlled trials (RCTs) for the prevention and treatment of periodontal disease

2. Selective outcome reporting in RCTs of oral medicine

3. Selective outcome reporting in RCTs of the management of oral mucositis in cancer patients

4. Core outcome set for traumatic dental injuries in children and adults.

Hopefully, the Rosetta mission will successfully deploy its lander Philae on the $12^{\text {th }}$ November and provide additional information about comets, and in due course the COMET initiative in dentistry will also deliver COS for a range of dental disease areas. While defining COS for dentistry will be one thing the next challenge will be ensuring that they are implemented.

1. Mulrow $C D$. The medical review article: state of the science. Ann Intern Med 1987; 106: 485-488.

2. Silagy CA. An analysis of review articles published in primary care journals. Fam Pract 1993; 10: 337-341.

3. Higgins JPT, Green S (editors). Cochrane Handbook for Systematic Reviews of Interventions Version 5.1.0 [updated March 2011]. The Cochrane Collaboration, 2011. Available from www.cochrane-handbook.org.

4. Systematic Reviews: CRD's guidance for undertaking systematic reviews in health care 2009. ISBN: 1900640473 Available from http://www.york.ac.uk/inst/crd/ index guidance.htm

5. Richards D. Time to up the research quality stakes. Evid Based Dent 2011; 12: 34-35.

6. Savage A, Eaton KA, Moles DR, Needleman I. A systematic review of definitions of periodontitis and methods that have been used to identify this disease. J Clin Periodontol 2009; 36: 458-467.

Evidence-Based Dentistry (2014) 15, 66. doi:10.1038/ sj.ebd.6401054 